\title{
Captures
}

Figures, théories et pratiques de l'imaginaire

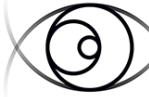

C A P T U R E S

\section{Volume 1 Numéro 1}

\section{Vincent Lavoie}

Volume 1, Number 1, 2016

Post-photographie?

URI: https://id.erudit.org/iderudit/1059818ar

DOI: https://doi.org/10.7202/1059818ar

See table of contents

Publisher(s)

Figura, Centre de recherche sur le texte et l'imaginaire

ISSN

2371-1930 (digital)

Explore this journal

Cite this document

Lavoie, V. (2016). Volume 1 Numéro 1. Captures, 1(1).

https://doi.org/10.7202/1059818ar

\section{Article abstract}

Attentive aux plus récentes manifestations de l'imaginaire contemporain et soucieuse d'en saisir les fondements historiques et théoriques, Captures est la toute nouvelle et première revue à comité de pairs de Figura, le Centre de recherche sur le texte et l'imaginaire. Sa création est source de grande fierté. Dédiée à la publication de travaux consacrés à l'analyse de problématiques liées aux figures, théories et pratiques de l'imaginaire, sans limitations territoriales ou...
Tous droits réservés (C) Vincent Lavoie, 2016

\section{(c) (i) $\Theta$}

This document is protected by copyright law. Use of the services of Érudit (including reproduction) is subject to its terms and conditions, which can be viewed online.

https://apropos.erudit.org/en/users/policy-on-use/
This article is disseminated and preserved by Érudit.

Érudit is a non-profit inter-university consortium of the Université de Montréal, Université Laval, and the Université du Québec à Montréal. Its mission is to promote and disseminate research.

https://www.erudit.org/en/ 
Version enrichie de cet article : http://revuecaptures.org/node/333

\section{Volume 1 Numéro 1}

\section{Vincent Lavoie}

Résumé :

Attentive aux plus récentes manifestations de l'imaginaire contemporain et soucieuse d'en saisir les fondements historiques et théoriques, Captures est la toute nouvelle et première revue à comité de pairs de Figura, le Centre de recherche sur le texte et l'imaginaire. Sa création est source de grande fierté. Dédiée à la publication de travaux consacrés à l'analyse de problématiques liées aux figures, théories et pratiques de l'imaginaire, sans limitations territoriales ou...

Attentive aux plus récentes manifestations de l'imaginaire contemporain et soucieuse d'en saisir les fondements historiques et théoriques, Captures est la toute nouvelle et première revue à comité de pairs de Figura, le Centre de recherche sur le texte et l'imaginaire Sa création est source de grande fierté. Dédiée à la publication de travaux consacrés à l'analyse de problématiques liées aux figures, théories et pratiques de l'imaginaire, sans limitations territoriales ou nationales, située à la croisée des études littéraires et cinématographiques, des sciences humaines, de l'histoire de l'art, des arts visuels et médiatiques, Captures se veut une pièce maîtresse de la diffusion internationale de la recherche menée au Centre. La revue exploite résolument les ressources du Web en publiant des numéros évolutifs, ouverts à des enrichissements continus et périphériques à chaque parution, et dont les contenus sont intégrés aux environnements de recherches et de connaissances mis en ligne par Figura. Assumant pleinement la mutabilité de son support, Captures aspire à renouveler les formes de la revue savante.

Ce premier numéro de la revue est bâti autour d'un dossier consacré à la post-photographie, une appellation non-consensuelle et labile, aux contours imprécis, qu'il convenait de discuter. À travers cette thématique, Captures fait le choix d'interroger les mutations touchant la photographie aujourd'hui, laquelle procède depuis ses origines historiques à la capture et à la saisie du réel, justement. Mais quelle prise sur le monde les images photographiques actuellement en usage nous offrent-elles, sachant la production de celles-ci incommensurable, leur mise en circulation tentaculaire et leur véracité souvent suspecte? II est apparu de circonstance, pour une revue nommée Captures, que sa naissance soit placée sous le signe d'une réflexion consacrée à cet archivage compulsif du présent. Offrant un espace à la recherche-création, le dossier « Postphotographie? » est assorti de «Contrepoints » présentant un ensemble d'œuvres en dialogue artistique avec le thème retenu. De plus, ce premier numéro accueille deux contributions inédites portant sur des considérations centrales au mandat de la revue. Son lancement coïncide avec la parution d'un article traitant 
d'une actualité artistique qui prend à bras le corps la question de l'imaginaire. Enfin, que Denis Farleyet Penelope Umbrico soient très chaleureusement remerciés de nous avoir confié leurs images pour l'élaboration de la signature visuelle de ce numéro.

Figura, le Centre de recherche sur le texte et l'imaginaire,souhaitait ajouter à son éventail de réalisations une revue à comité de pairs porteuse de sa mission. Cela est désormais chose faite avec la création de Captures.

Vincent Lavoie

Directeur 